\title{
Implementation of the $\in$ - Constraint Method in Special Class of Multi-objective Fuzzy Bi-Level Nonlinear Problems
}

\author{
Azza H. Amer \\ Department of Mathematics, Faculty of Science, Helwan University, Cairo, Egypt \\ amer_dr_azza@yahoo.com
}

\begin{abstract}
Geometric programming problem is a powerful tool for solving some special type nonlinear programming problems. In the last few years we have seen a very rapid development on solving multiobjective geometric programming problem. A few mathematical programming methods namely fuzzy programming, goal programming and weighting methods have been applied in the recent past to find the compromise solution. In this paper, $\in$-constraint method has been applied in bi-level multiobjective geometric programming problem to find the Pareto optimal solution at each level. The equivalent mathematical programming problems are formulated to find their corresponding value of the objective function based on the duality theorem at eash level. Here, we have developed a new algorithm for fuzzy programming technique to solve bi-level multiobjective geometric programming problems to find an optimal compromise solution. Finally the solution procedure of the fuzzy technique is illustrated by a numerical example.
\end{abstract}

Keywords: Geometric programming; $\in$-constraint method; Fuzzy programming; Duality theorem; Bi-level programming; Pareto optimal solution.

\section{Introduction}

Various mathematical programming methods have been formulated to solve many real world problems. Since late 1960's, geometric programming (GP) has been known and used in various fields (like operations research (OR), Engineering sciences etc.). It's founders include Duffin et al., (1967) and Peterson (2001). GP is useful in the study of a variety of optimization problems and it is technique developed for solving algebraic nonlinear programming problems subject to linear or nonlinear constraints.

Today, most of the real world decision making problems in economic, environmental, social, and technical areas are multidimensional and multiobjective ones. Multiobjective optimization problems differ from single objective optimization problem. The purpose of multiobjective problems (MOP) in the mathematical programming (MP) framework is to optimize the different objective problems simultaneously subject to a set of system constraints (Islam and Roy, 2005; Islam, 2010; Bazikar and Sarj, 2014; Bit, 1998). However, the decision makers (DMs) are always in search of a most compromise solution that could optimize all objective functions, known as Pareto optimal solution (Ojha and Biswal, 2014; Ojha and Ota, 2014). According to Hwang and Masud (Hwang and Masud, 1979) multiobjective optimization method can be classified into three categories such as priori method, interactive method and generation method. The priori method is based on the goals or weights which are to be set by the decision maker before solution process which is a difficult task in the part of decision maker for obtaining compromise solution. Interactive method are used to find the solution interactively. The generating methods are less popular due to their computational effort and is used to find Pareto optimal solution (Chankong and Haimes, 1983; Mavrotas, 2009). So, this research is considered the main problem in the form of bi-level geometric programming problem (BLGPP). 
Bi-level programming problems (BLPP) form an important class optimization problems involving hierarchical decision making. Processes where the Upper level (UL) decision maker anticipates the responses from the Lower level (LL) and proceeds with optimizing its own objective. Their origin traces back to the Stackelberg competition model in economics (Von-Stackelberg, 1952). In recent years, the optimization problems with hierarchical structures have attracted a great amount of interests among researchers in OR, economics and applied mathematics (Zhanf, 2003; Bard, 1999; Vincente and Calamai, 1993). In this paper we use Lee and Hus-Shihshih (2001) work to solve BLGPP. Also we extend (Ojha and Biswal, 2014; Ojha and Ota, 2014) to solve bi-level multiobjective geometric programming problem (BLMOGPP)as: First, at each level we have applied $\in$-constraint method to solve a special class of multiobjective geometric programming problems (MOGPP) where $\in$-constraint method is optimize one of the objective function at a time where other objectives are kept in the constraint part of the model. This method is found more suitable than the other generating methods used for obtaining Pareto optimal solution. After obtaining lower and upper bounds of each objective function we find the Pareto optimal solution. Also, we have used fuzzy programming problem due to Zimmermann (1990) based on the concept given by Bellman and Zadeh (1970) at each level to find the lower and upper bounds of each objective function. It has been successfully applied to solve various types of multiobjective decision making problems. Second, a develop new fuzzy programming (FP) technique have been applied in BLMOGPP to find the optimal value of the functions due to the lower and upper bounds of the previous step (Yang and Cao, 2010; Zhou et al., 2016) through given a step - by - step new algorithm to the FPBLMOGPP. Finally an illustralive example is presented to find the Pareto optimal solution using $\in$-constraint method and fuzzy programming method to BLMOGPP.

\section{Bi-level Multiobjective Geometric Programming Problem (BLMOGPP)}

In a bi-level problem, multiple decision entities may exist at each level, and this is known as a bi-level multi-leader and /or multi-follower decision problem. A bi-level multiobjective geometric programming problem can be stated as (Lu et al., 2016):

For $x \in X \subset R^{p}, y \in Y \subset R^{q},(X, Y) \subset R^{L=p+q}$ so as to

$$
\min _{x \in X} F(x, y)=\left(F_{1}(x, y), F_{2}(x, y), \ldots, F_{M}(x, y)\right)
$$

s.t.

$$
\begin{aligned}
& G(x, y) \leq 1 \\
& (x, y)>0
\end{aligned}
$$

where, for each $x$ given by the first level, $y$ solves

(lower level):

$$
\min _{y \in Y} f(x, y)=\left(f_{1}(x, y), f_{2}(x, y), \ldots, f_{N}(x, y)\right)
$$

s.t.

$$
\begin{aligned}
& g(x, y) \leq 1 \\
& (x, y)>0
\end{aligned}
$$


where $x, y$ are decision variables of the Upper level and the Lower level respectively; $F_{i}, f_{j}: R^{p} \times R^{q} \rightarrow R^{L} \quad, i=1,2, \ldots, M, j=1,2, \ldots, N$ are the conflicting objective functions of the Upper level and the Lower level respectively; $G: R^{p} \times P^{q} \rightarrow R^{m}, g: R^{p} \times R^{q} \rightarrow R^{n}$ are the constraint conditions of the Upper level and the Lower level respectively. The sets $\mathrm{X}$ and $\mathrm{Y}$ place additional restrictions on the decision variables, such as upper and lower bounds or integrality requirements. Also

$$
\begin{array}{lll}
F_{i}(x, y)=\sum_{t=1}^{T_{i}} c_{i t} \prod_{b=1}^{\mathrm{q}} \prod_{r=1}^{\mathrm{p}} x_{r}^{a_{i t r}} y_{b}^{e_{i t b}} & , i \in\{1,2, \ldots, M\}, \\
f_{j}(x, y)=\sum_{t=1}^{T_{j}} c_{j t} \prod_{b=1}^{\mathrm{q}} \prod_{r=1}^{\mathrm{p}} x_{r}^{a_{j t r}} y_{b}^{e_{j t b}} & , j \in\{1,2, \ldots, N\}, \\
G_{i^{\prime}}(x, y)=\sum_{t=1}^{T_{i^{\prime}}} c_{i^{\prime} t} \prod_{b=1}^{\mathrm{q}} \prod_{r=1}^{\mathrm{p}} x_{r}^{a_{i^{\prime} t r}} y_{b}^{e_{i^{\prime} t b}} & , i^{\prime}=1,2, \ldots, m, \\
g_{j^{\prime}}(x, y)=\sum_{t=1}^{T_{j^{\prime}}} c_{j^{\prime} t} \prod_{b=1}^{\mathrm{q}} \prod_{r=1}^{\mathrm{p}} x_{r}^{a_{j^{\prime} t r}} y_{b}^{e_{j^{\prime} t b}} & , j^{\prime}=1,2, \ldots, n,
\end{array}
$$

where $c_{i t}>0$ for all $i$ and $t, c_{j t}>0$ for all $j$ and $t, c_{i^{\prime} t}>0$ for all $i^{\prime}$ and $t, c_{j^{\prime} t}>0$ for all $j^{\prime}$ and $t ; a_{i t r}, a_{j t r}, a_{i^{\prime} t r}$ and $a_{j^{\prime} t r}$ are real numbers for all $i, j, t, r, i^{\prime}, j^{\prime} ; T_{i}$ is the number of terms present in the $i$ th objective function $F_{i}(x, y), i=1,2, \ldots M ; T_{j}$ is the number of terms present in the $j$ th objective function $f_{j}(x, y), j=1,2, \ldots, N ; T_{i^{\prime}}$ is the number of terms present in the $i^{\prime}$ th constraint, $i^{\prime}=1,2, \ldots, m$ and $T_{j^{\prime}}$ is the number of terms present in the $j^{\prime}$ th constraint, $j^{\prime}=1,2, \ldots, n$.

In the above BLMOGPP from (2.1)-(2.6) there are $(\mathrm{M}+\mathrm{N})$ number of minimization type objective functions, $(m+n)$ number of inequality type constraints and $L=p+q$ number of strictly positive decision variables. Both upper level problem (2.1) - (2.3) and lower level problem (2.4) - (2.6) is considered as a vector minimum problem. It is assumed that the two problems have an optimal compromise solution.

\section{Upper Level Muliobjective Geometric Programming Problem (ULMOGPP) with $\in$ - Constraint Method}

The $\in$ - constraint method is proposed by Haimes et al. (1971) for generating Pareto optimal solutions. Since MOGPP is special type of vector minimum problem, the $\in$ constraint method is applicable to the present form. It is assumed that the present problem from (2.1) - (2.3) and the problem (2.4) - (2.6) are feasibles and have optimal compromise solutions.

The present ULMOGPP (2.1) - (2.3) can be redefined as a single objective geometric programming Problem (GPP) by using $\in$ - constraint method as:

(ULGPP): 


$$
\min _{x \in X} F_{i}(x, y)=\sum_{t=1}^{T_{i}} c_{i t} \prod_{b=1}^{q} \prod_{r=1}^{p} x_{r}^{a_{i t r}} y_{b}^{e_{i t b}} \quad, i \in\{1,2, \ldots, M\},
$$

s.t.

$$
F_{h}(x, y)=\sum_{t=1}^{T_{h}} c_{h t} \prod_{b=1}^{q} \prod_{r=1}^{p} x_{r}^{a_{h t r}} y_{b}^{e_{h t b}} \leq \epsilon_{h} \quad, h=1,2, \ldots, M, h \neq i
$$

and

$$
\begin{array}{ll}
G_{i^{\prime}}(x, y)=\sum_{t=1}^{T_{i^{\prime}}} c_{i^{\prime} t} \prod_{b=1}^{q} \prod_{r=1}^{p} x_{r}^{a_{i^{\prime} t r}} y_{b}^{e_{i^{\prime} t b}} \leq 1 & , i^{\prime}=1,2, \ldots, m, \\
x_{r}>0 \quad, r=1,2, \ldots, p \quad, y_{b}>0 & , b=1,2, \ldots, q,
\end{array}
$$

where $\in_{h}>0$, for all $h$.

Here we have $\mathrm{M}$ number of single objective geometric programming problem (GPP 1 ). This problem can be simplified as:

\section{(GPP1):}

$$
\min _{x \in X} \bar{F}_{i}(x, y)=\sum_{t=1}^{T_{i}} \bar{c}_{i t} \prod_{b=1}^{q} \prod_{r=1}^{p} x_{r}^{a_{i t r}} y_{b}^{e_{i t b}} \quad, i \in\{1,2, \ldots, M\}
$$

s.t.

$$
\begin{array}{lll}
\text { i.e. } \quad & \bar{F}_{h}(x, y)=\sum_{t=1}^{T_{h}} \bar{c}_{h t} \prod_{b=1}^{q} \prod_{r=1}^{p} x_{r}^{a_{h t r}} y_{b}^{e_{h t b} \leq 1}, h=1,2, \ldots, M, h \neq i, \\
\bar{G}_{i^{\prime}}(x, y)=\sum_{t=1}^{T_{i^{\prime}}} \bar{c}_{i^{\prime} t} \prod_{b=1}^{q} \prod_{r=1}^{p} x_{r}^{a_{i^{\prime} t r}} y_{b}^{e_{i^{\prime} t b}} \leq 1 & , i^{\prime}=1,2, \ldots, m, \\
x_{r}>0, r=1,2, \ldots, p, \quad y_{b}>0 & , b=1,2, \ldots, q,
\end{array}
$$

where $\bar{c}_{h t}=\frac{c_{h t}}{\in_{h}}$ for all h, t, $h \neq i$,

$$
\bar{c}_{i t}=c_{i t}, \bar{F}_{i}(x, y)=F_{i}(x, y) \text { for } i \in\{1,2, \ldots, M\} \text {. }
$$

The $\mathrm{GPP}_{1}$ has $[(\mathrm{M}-1)+\mathrm{m}]$ constraints and $[\mathrm{L}=\mathrm{p}+\mathrm{q}]$ number of decision variables.

The degree of difficulty of this problem can be defined as:

$$
\bar{d}=\sum_{i=1}^{M} T_{i}+\sum_{i^{\prime}=1}^{m} T_{i^{\prime}}-L-1
$$

The solution procedure for a GP may be categorized as of two types. It is either primal based algorithms that directly solve nonlinear primal problem, or dual based algorithms that solve the equivalent linear constraint dual program (Peterson, 2001). The dual form of GPP plays an important role in solving complex types of single and multiobjective 
optimization problems. It has been verified, if primal program of the problem is super consistent and attains its constrained minimum value, then its corresponding dual program is consistent and attaines its corresponding maximum value where the optimal values due to primal and dual program are same. The dual program of ULMOGPP can be formed as:

\subsection{Dual Program of ULMOGPP}

The dual program of $\mathrm{GPP}_{1}$ can be stated as:

$$
\max V(w)=\prod_{i=1}^{M} \prod_{t=1}^{T_{i}}\left(\frac{\bar{c}_{i t}}{w_{i t}}\right)^{w_{i t}} \prod_{i^{\prime}=1}^{m} \prod_{t=1}^{T_{i^{\prime}}}\left(\frac{c_{i^{\prime} t}}{w_{i^{\prime} t}}\right)^{w_{i^{\prime} t}} \prod_{i=1}^{M}\left(\lambda_{i}\right)^{\lambda_{i}} \prod_{i^{\prime}=1}^{m}\left(\lambda_{i^{\prime}}\right)^{\lambda_{i^{\prime}}}
$$

s.t.

$$
\begin{aligned}
& \sum_{t=1}^{T_{h}} w_{h t}=\lambda_{i} \quad, i=1,2, \ldots, M, i \neq h, \\
& \sum_{t=1}^{T_{i}} w_{i t}=\lambda_{i}=1 \quad, \quad \text { (normality condition), } \\
& \sum_{t=1}^{T_{i^{\prime}}} w_{i^{\prime} t}=\lambda_{i^{\prime}} \quad, i^{\prime}=1,2, \ldots, m, \\
& \sum_{i=1}^{M} \sum_{t=1}^{T_{i}} a_{i t r} w_{i t}+\sum_{i^{\prime}=1}^{m} \sum_{t=1}^{T_{i^{\prime}}} a_{i^{\prime} t r} w_{i^{\prime} t}=0 \quad, r=1,2, \ldots, P, \\
& \sum_{i=1}^{M} \sum_{t=1}^{T_{i}} e_{i t b} w_{i t}+\sum_{i^{\prime}=1}^{m} \sum_{t=1}^{T_{i^{\prime}}} e_{i^{\prime} t b} w_{i^{\prime} t}=0 \quad, b=1,2, \ldots, q, \\
& w_{i t} \geq 0, \text { for all } i, t, w_{i^{\prime} t} \geq 0, \text { for all } i^{\prime}, t .
\end{aligned}
$$

This dual problem can be solved by using duality theory of GPP [24].

\section{Lower Level Multiobjective Geometric Programming Problem (LLMOGPP) with $\in$ - Constraint Method}

LLMOGPP as in (2.4) - (2.6) can be redefined as a single objective GPP by using $\in$ constraint method as:

\section{(LLGPP):}

$$
\min _{y \in Y} f_{j}(x, y)=\sum_{t=1}^{T_{j}} c_{j t} \prod_{b=1}^{q} \prod_{r=1}^{p} x_{r}^{a_{j t r}} y_{b}^{e_{j t b}} \quad, \quad j \in\{1,2, \ldots, N\},
$$

s.t.

$$
f_{s}(x, y)=\sum_{t=1}^{T_{s}} c_{s t} \prod_{b=1}^{q} \prod_{r=1}^{p} x_{r}^{a_{s t r}} y_{b}^{e_{s t b}} \leq \in_{s}, s=1,2, \ldots, N, s \neq j,
$$

and 


$$
\begin{aligned}
& g_{j^{\prime}}(x, y)=\sum_{t=1}^{T_{j^{\prime}}} c_{j^{\prime} t} \prod_{b=1}^{q} \prod_{r=1}^{p} x_{r}^{a_{j^{\prime} t r}} y_{b}^{e_{j^{\prime} t b}} \leq 1, \quad j^{\prime}=1,2, \ldots, n, \\
& x_{r}>0, r=1,2, \ldots, p, \quad y_{b}>0 \quad, \quad b=1,2, \ldots, q,
\end{aligned}
$$

where $\in_{s}>0$, for all $s$.

Here we have $\mathrm{N}$ number of single objective geometric programming problem $\left(\mathrm{GPP}_{2}\right)$. The above problem can be simplified as:

$\left(\mathbf{G P P}_{2}\right)$ :

$$
\min _{y \in Y} \hat{f}_{j}(x, y)=\sum_{t=1}^{T_{j}} \hat{c}_{j t} \prod_{b=1}^{q} \prod_{r=1}^{p} x_{r}^{a_{j t r}} y_{b}^{e_{j t b}} \quad, \quad j \in\{1,2, \ldots, N\},
$$

s.t.

$$
\hat{f}_{s}(x, y)=\sum_{t=1}^{T_{s}} \frac{c_{s t}}{\in_{s}} \prod_{b=1}^{q} \prod_{r=1}^{p} x_{r}^{a_{s t r}} y_{b}^{e_{s t b}} \leq 1, \quad s=1,2, \ldots, N, s \neq j,
$$

i.e. $\hat{f}_{s}(x, y)=\sum_{t=1}^{T_{s}} \hat{c}_{s t} \prod_{b=1}^{q} \prod_{r=1}^{p} x_{r}^{a_{s t r}} y_{b}^{e_{s t b}} \leq 1, s=1,2, \ldots, N, s \neq j$,

$$
\begin{aligned}
& \hat{g}_{j^{\prime}}(x, y)=\sum_{t=1}^{T_{j^{\prime}}} \hat{c}_{j^{\prime} t} \prod_{b=1}^{q} \prod_{r=1}^{p} x_{r}^{a_{j^{\prime} t r}} y_{b}^{e_{j^{\prime} t b}} \leq 1, j^{\prime}=1,2, \ldots, n, \\
& x_{r}>0, r=1,2, \ldots, p \quad, \quad y_{b}>0 \quad, b=1,2, \ldots, q,
\end{aligned}
$$

where $\underset{s t}{\hat{c}}=\frac{c_{s t}}{\in_{s}}$ for all $s, t, \quad s \neq j$,

$$
\hat{c}_{j t}=c_{i t}, \hat{f}_{j}(x, y)=f_{j}(x, y) \text { for } j \in\{1,2, \ldots, N\} \text {. }
$$

The $\mathrm{GPP}_{2}$ has $[(\mathrm{N}-1)+\mathrm{n}]$ constraints and $[\mathrm{L}=\mathrm{p}+\mathrm{q}]$ number of decision variables. The degree of difficulty of this problem is:

$$
\hat{d}=\sum_{j=1}^{N} T_{j}+\sum_{j^{\prime}=1}^{n} T_{j^{\prime}}-q-1
$$

\subsection{Dual Program of LLMOGPP}

The duel program of $\mathrm{GPP}_{2}$ can be stated as:

$$
\max V^{\prime}(w)=\prod_{j=1}^{N} \prod_{t=1}^{T_{j}}\left(\frac{\hat{c}_{j t}}{w_{j t}}\right)^{w_{j t}} \prod_{j^{\prime}=1}^{n} \prod_{t=1}^{T_{j^{\prime}}}\left(\frac{\hat{c}_{j^{\prime} t}}{w_{j^{\prime} t}}\right)^{w_{j^{\prime} t}} \prod_{j=1}^{N}\left(\lambda_{j}\right)^{\lambda_{j}} \prod_{j^{\prime}=1}^{n}\left(\lambda_{j^{\prime}}\right)^{\lambda_{j^{\prime}}}
$$

s.t.

$$
\sum_{t=1}^{T_{s}} w_{s t}=\lambda_{j} \quad, j=1,2, \ldots, N, \quad j \neq s
$$




$$
\begin{aligned}
& \sum_{t=1}^{T_{j}} w_{j t}=\lambda_{j}=1 \quad, \quad \text { (normality condition), } \\
& \sum_{t=1}^{T_{j^{\prime}}} w_{j^{\prime} t}=\lambda_{j^{\prime}} \quad, \quad j^{\prime}=1,2, \ldots, n, \\
& \sum_{j=1}^{N} \sum_{t=1}^{T_{j}} a_{j t r} w_{j t}+\sum_{j^{\prime}=1}^{n} \sum_{t=1}^{T_{j^{\prime}}} a_{j^{\prime} t r} w_{j^{\prime} t}=0 \quad, r=1,2, \ldots, P, \\
& \sum_{j=1}^{N} \sum_{t=1}^{T_{j}} e_{j t b} w_{j t}+\sum_{j^{\prime}=1}^{n} \sum_{t=1}^{T_{j^{\prime}}} e_{j^{\prime} t b w_{j^{\prime} t}=0,}, b=1,2, \ldots, q, \\
& w_{j t} \geq 0, \text { for all } j, t, \quad w_{j^{\prime} t} \geq 0, \text { for all } j^{\prime}, t .
\end{aligned}
$$

This dual program can be solved by using duality theory of GPP [24].

\section{Fuzzy Decision Models for BLMOGPP}

To solve BLMOGPP by adopting, the leader-follower Stackelberg game, and the wellknown fuzzy decision model of Bellman and Zadeh (1970), who have introduced fuzzy set theory in 1965 which is generalization of classical set theory to understand the uncertainty and vagueness in the complexity of the problems. Fuzzy programming problem (FPP) has been successfully applied to solve various types of multiobjective decision making problems such as engineering design and maintenance, production planning and control, transportation, water resource management, managerial decision making and scheduling program. A fuzzy set is associated with its membership function which is defined from its elements to the interval [0,1] plays an important role in solving multiobjective decision making problems. There are several type of fuzzy membership functions, so a membership function is to be selected to solve the real world multiobjective mathematical programming problems is a suitable. Also we get the satisfactory solution that is acceptable to Upper level decision maker (ULDM), and then give the ULDM decision variables and goals with some information to the lower level decision maker (LLDM) for him/her to seek the satisfactory solution and to arrive at the solution which is closest to the satisfactory solution of the ULDM. This due to, the LLDM should not only optimize his/her objective functions but also try to satisfy the ULDM's goals and preference as much as possible (Lee and Hsu-Shihshih, 2001). In this way, the solution method simplifies a BLMOGPP by transforming it into separate MOGPP at Upper and Lower levels, by that means the difficulty associated with nonconvexity to arrive at an optimal solution, is avoided (Abo-Sinna, 2001; Osman et al., 2004) The following algorithm steps are used to solve a multiobjective optimization problem with a linear member functions by geometric programming technique to find an optimal compromise solution. 


\subsection{Fuzzy Programming Method for ULMOGPP (FPULMOGPP)}

\section{Algorithm (1):}

Step 1: First, choose one of the objective function $F_{i}(x, y), i=1,2, \ldots, M$, and solve it as a single objective geometric programming problem subject to the constraints (2.2) and (2.3) by using GP algorithm (Beightler and Philips, 1976)). Let $\left(X_{i}, Y_{i}\right), i=1, \ldots, M$ be the respective ideal solution for $\mathrm{M}$ different GP problems. It is assumed that at least two of these ideal solution are different $\left(F_{i}(x, y), i=1,2, \ldots, M\right)$ and has the different bound values. If all the optimal solution $\left(X_{i}, Y_{i}\right)=\left(X^{*}, Y^{*}\right), i=1,2, \ldots, M$ are same then stop and $\left(X^{*}, Y^{*}\right)$ is the optimal compromise solution. Otherwise go to step 2 .

Step 2: Evaluate all these $\mathrm{M}$ objective functions $\left(F_{i}(x, y), i=1,2, \ldots, M\right)$ at all these $\mathrm{M}$ ideal solutions $\left(X_{i}, Y_{i}\right), i=1,2, \ldots, M$.

Step 3: Find the best value $l_{i}$ (minimum value) and the worst value $u_{i}$ (maximum value) at each objective function $F_{i}(x, y)$ such that,

$l_{i} \leq F_{i}(x, y) \leq u_{i} \quad, i=1,2, \ldots, M$.

Step 4: Let $\epsilon_{i}$ be the point in the interval such that, $l_{i} \leq \epsilon_{i} \leq u_{i}, i=1,2, \ldots, M$ and change the value of $\epsilon_{i}$ in the interval $\left[l_{i}, u_{i}\right]$, we can generate a set of Pareto optimal solution by using $\mathrm{GPP}_{1}$, see section 3.1.

Step 5: Goals and tolerances can be reasonably set for individual solutions and the differences of the best and worst solutions, respectively. This data can then be formulated as the following membership functions of fuzzy set theory (Zimmermann, 1990):

$\mu_{F_{i}}\left(F_{i}(x, y)= \begin{cases}1 & \text { if } F_{i}(x, y) \leq l_{i}, \\ \frac{U_{i}-F_{i}(x, y)}{u_{i}-l_{i}}, & \text { if } l_{i} \leq F_{i}(x, y) \leq u_{i}, \\ 0 & \text { if } F_{i}(x, y) \geq u_{i}\end{cases}\right.$

where $l_{i} \neq u_{i}, i=1,2, \ldots, M$.

If $l_{i}=u_{i}$, then define $\mu_{i}(x, y)=1$ for any value of $i$.

Step 6: Obtain the solution of FPULMOGPP by solving the following Tchebycheff problem (Lee and Hsu-Shihshih, 2001):

$\operatorname{Max} \alpha$

s.t.

$\frac{u_{i}-F_{i}(x, y)}{u_{i}-l_{i}} \geq \alpha \quad, i=1,2, \ldots, M$, 


$$
\begin{array}{ll}
G(x, y) & \leq 1, \\
(x, y) & >o, \\
\alpha \in[0,1] . &
\end{array}
$$

whose solution is assumed to be $X^{U}, Y^{U}, F_{i}^{U}, i=1,2, \ldots, M, \alpha^{U}$ (satisfactory level). Further the inequality (5.4) can be represented as:

$$
F_{i}(x, y)+\left(u_{i}-l_{i}\right) \alpha \leq u_{i}, i=1,2, \ldots, M
$$

Also $\quad l_{i} \leq \in_{i} \leq u_{i} \quad, i=1,2, \ldots, M$.

\subsection{Fuzzy Programming Method for LLMOGPP (FPLLMOGPP)}

\section{Algorithm (2):}

Step 1: In the same way, the LLMOGPP independently solves

$$
\min f_{j}(x, y) \quad, j=1,2, \ldots N
$$

s.t.

$$
\begin{aligned}
& g(x, y) \leq 1 \\
& (x, y)>0
\end{aligned}
$$

Step 2: $\quad$ Find individual best value $l_{j}$ (minimum value) and individual worst value $u_{j}$ (maximum value) at each objective function $f_{j}(x, y)$ such that,

$$
l_{j} \leq f_{j}(x, y) \leq u_{j} \quad, j=1,2, \ldots, N
$$

Step 3: Let $\in_{j}$ be the point in the interval such that, $l_{j} \leq \in_{j}(x, y) \leq u_{j}, j=1,2, \ldots, N$ and change the value of $\epsilon_{j}$ in the interval $\left[l_{j}, u_{j}\right]$, we can generate a set of Pareto optimal solution by using $\mathrm{GPP}_{2}$, see section 4.1 .

Step 4: Formulate the following membership functions of fuzzy theory (Zimmermann, 1990) by using the above information as:

$$
\mu_{f_{j}}\left(f_{j}(x, y)\right)= \begin{cases}1 \quad, & \text { if } f_{j}(x, y) \leq l_{j} \\ \frac{u_{j}-f_{j}(x, y)}{u_{j}-l_{j}}, & \text { if } l_{j} \leq f_{j}(x, y) \leq u_{j} \\ 0 \quad, & \text { if } f_{j}(x, y) \geq u_{j}\end{cases}
$$

where $l_{j} \neq u_{j}, j=1,2, \ldots, N$.

If $l_{j}=u_{j}$. Then define $\mu_{j}(x, y)=1$ for any value of $j$. 
Step 5: Obtain the solution of FPLLOGPP by solving the following Tchebycheff problem (Lee and Hsu-Shihshih, 2001):

$$
\operatorname{Max} \beta
$$

s.t.

$$
\begin{aligned}
& \frac{u_{j}-f_{j}(x, y)}{u_{j}-l_{j}} \geq \beta \quad, j=1,2, \ldots, N, \\
& g(x, y) \leq 1, \\
& (x, y)>0, \\
& \beta \in[0,1] .
\end{aligned}
$$

whose solution is assumed to be $X^{L}, Y^{L}, F_{j}^{L}, j=1,2, \ldots, N, \beta^{L}$ (satisfactory level). Further the inequality (5.13) can be represented as:

$$
f_{j}(x, y)\left(u_{j}-l_{j}\right) \beta \leq u_{j} \quad, j=1,2, \ldots, N
$$

Also, $\quad l_{j} \leq \in_{j} \leq u_{j}$

$$
j=1,2, \ldots, N
$$

\subsection{Fuzzy Programming Method for BLMOGPP (FPBLMOGPP)}

\section{Algorithm (3):}

Step 1: Find the above solution of FPULMOGPP and the solution of FPLLMOGPP by using an algorithm (1) and an algorithm (2) respectively. However two solutions are usually different because of confilicts of nature between two levels objective function. The FPULMOGPP knows that using the optimal decision $X^{U}$ as a control factor for FPLLMOGPP is not practical. It is more reasonable to have some tolerance that gives the FPLLMOGPP an extent feasible region to search for his/her optimal solution, and also reduce searching time or interactions. In this way, the range of the decision variable $\mathrm{X}$ should be around $X^{U}$ with its maximum tolerances $\mathrm{k}$.

Step 2: Formulate the following membership function which specify $X^{U}$ as:

$$
\mu_{X}(X)=\left\{\begin{array}{cc}
\frac{\left[X-\left(X^{U}-k\right)\right]}{k} & , \text { if } X^{U}-k \leq X \leq X^{U}, \\
k & , \text { if } X^{U} \leq X \leq X^{U}+k, \\
0 & , \quad \text { Otherwise }
\end{array}\right.
$$

where $X^{U}$ is the most preferred solution (Zimmermann, 1990). 
Step 3: Consider the following membership function of the FPULMOGPP at all $l_{i} \leq F_{i} \leq u_{i} \quad, i=1,2, \ldots, M$, as:

$\mu_{F_{i}}\left(F_{i}(x, y)\right)= \begin{cases}1 & \text { if } F_{i}(x, y) \leq l_{i} \\ \frac{u_{i}^{\prime}-F_{i}(x, y)}{u^{\prime}-l_{i}}, & \text { if } l_{i} \leq(x, y) \leq u_{i}^{\prime} \\ 0 & \text { if } F_{i}(x, y) \geq u_{i}^{\prime}\end{cases}$

where $u_{i}^{\prime}=F_{i}\left(X^{L}, Y^{L}\right)$.

Step 4: Formulate the following membership functions of FPLLMOGPP for his/her goals as:

$$
\mu_{f_{i}}\left(f_{j}(x, y)\right)= \begin{cases}1 \quad & \text { if } f_{j}(x, y) \geq l_{j} \\ \frac{\hat{u}_{j}-f_{j}(x, y)}{\hat{u}_{j}-l_{j}}, & \text { if } l_{j} f_{j}(x, y) \leq \hat{u}_{j} \\ 0 \quad, & \text { if } f_{j}(x, y) \geq \hat{u}_{j}\end{cases}
$$

where $\hat{u}_{j}=f_{j}\left(X^{U}, Y^{U}\right)[16]$.

Step 5: Generate the satisfactory solution which is also a Pareto optimal solution with an overall satisfaction for both decision makers (DMs) by solving the following Tchebycheff problem as follows (Lee and Hsu-Shihshih, 2001; Sakawa, 1993):

$\operatorname{Max} \delta$

s.t.

$$
\begin{aligned}
& {\left[X-\left(X^{U}-k\right)\right] / k \geq \delta I,} \\
& {\left[\left(X^{U}+k\right)-X\right] / k \geq \delta I,} \\
& \mu_{F_{i}}\left(F_{i}(x, y)\right)=\left[u_{i}^{\prime}-F_{i}(x, y)\right] /\left[u_{i}^{\prime}-l_{i}\right] \geq \delta, i=1,2, \ldots, M, \\
& \left.\mu_{f_{j}}\left(f_{j}(x, y)\right)=\mid \hat{u}_{j}-f_{j}(x, y)\right] /\left[\hat{u}_{j}-l_{j}\right] \geq \delta, \quad j=1,2, \ldots, N, \\
& G(x, y) \leq 1, \\
& g(x, y) \leq 1, \\
& (x, y) \quad>0, \\
& \delta \in[0,1] .
\end{aligned}
$$

where $\delta$ is the overall satisfaction, and I is a column vector with all elements equal to one's (ls) and the same direction as X. The problem from $(5.22)-(5.30)$ is actually a fuzzy problem by Sakawa (1993). 


\section{Convergence of the Optimal Solution}

By solving problem (5.22) - (5.30), if the ULMOGPP and $l_{i} \leq \in_{i} \leq u_{i}, i=1,2, \ldots, M$, are satisfied with this solution, then a satisfactory solution is reached. Other, he/she should provide new membership functions for the control variable and objective to LLMOGPP until a satisfactory solution is reached. In general, combined with set of control decision and objectives with tolerance, the solution of problem (5.22) - (5.30) becomes a Pareto optimal (satisfactory) solution for ULMOGPP from (2.1) - (2.3) and LLMOGPP from (2.4) - (2.6).

An illustrative example is presented to find the Pareto optimal solution using $\in$ constraint and fuzzy programming method for BLMOGPP.

\section{Numerical Example}

To demonstrate the solution method for BLMOGPP, let us consider the following example.

Find $x=\left(x_{1}, x_{2}, x_{3}\right)$ so as to ULMOGPP as:

$$
\min _{x_{1}} F(x)=\left\{\begin{array}{l}
\min _{x_{1}}: F_{1}(x)=20 x_{1}^{-1} x_{2}^{-3} x_{3}^{-5}+60 x_{1}^{-1} x_{2}^{-1}, \\
\min _{x_{1}}: F_{2}(x)=50 x_{1}^{-1} x_{2}^{-2} x_{3}^{-2}+60 x_{1}^{3} x_{2}^{-2} x_{3}^{-3}
\end{array}\right.
$$

s.t.

$$
\begin{aligned}
& G_{1}(x)=x_{1} x_{2} x_{3}^{2}+x_{2} x_{3} \leq 3, \\
& x=\left(x_{1}, x_{2}, x_{3}\right)>0
\end{aligned}
$$

where $x_{2}, x_{3}$ solves LLMOGPP as:

$$
\min _{x_{2}, x_{3}} f(x)=\left\{\begin{array}{l}
\min _{x_{2}, x_{3}}: f_{1}(x)=x_{1}^{-2}+0.25 x_{2}^{2} x_{3}^{-1} \\
\min _{x_{2}, x_{3}}: f_{2}(x)=2 x_{1}^{-1} x_{2}^{-1} x_{3}^{-1}+2 x_{1} x_{2}
\end{array}\right.
$$

s.t.

$$
\begin{aligned}
& g_{1}(x)=\frac{3}{4} x_{1}^{2} x_{2}^{-2}+\frac{3}{8} x_{2} x_{3}^{2} \leq 1 \\
& x=\left(x_{1}, x_{2}, x_{3}\right)>0
\end{aligned}
$$

First, Using the steps of algorithm (1) in FPULMOGPP as:

1. Finds individual optimal solution for each objective functions (6.1) and (6.2) as:

$$
\min _{X_{1}} F_{1}(x)=20 x_{1}^{-1} x_{2}^{-3} x_{3}^{-5}+60 x_{1}^{-1} x_{2}^{-1}
$$

s.t.

$$
\begin{aligned}
& G_{1}(x)=x_{1} x_{2} x_{3}^{2}+x_{2} x_{3} \leq 3 \\
& \left(x_{1}, x_{2}, x_{3}\right)>0
\end{aligned}
$$

Local optimal solution is: 
$\min F_{1}(x)=20.66567$ and $\left(x_{1}=1.952, x_{2}=4.4619, x_{3}=0.3842\right)$

Also

$\min _{X_{1}} F_{2}(x)=50 x_{1}^{-1} x_{2}^{-3} x_{3}^{-2}+60 x_{1}^{3} x_{2}^{-2} x_{3}^{-3}$

s.t.

$$
\begin{aligned}
& G_{1}(x)=x_{1} x_{2} x_{3}^{2}+x_{2} x_{3} \leq 3, \\
& \left(x_{1}, x_{2}, x_{3}\right)>0
\end{aligned}
$$

Local optimal solution is:

$\min F_{2}(x)=18.20745$ and $\left(x_{1}=0.47087, x_{2}=11.4422, x_{3}=0.23597\right)$

2. Using the local solution of $\left(\mathrm{p}_{1}\right)$ in (6.2) and the local solution of $\left(\mathrm{p}_{2}\right)$ in (6.1) in order to find the lower bound $l_{i}$ and upper bound $u_{i}$ of the function $F_{i}, i=1,2$.

$l_{1}=20.66567<F_{1}<49.89122=u_{1} ;$ and $l_{2}<18.20745<F_{2}<404.0162=u_{2}$. That is $20.66567<\epsilon_{1}<49.89122$; and $18.20745<\epsilon_{2}<404.0162$.

3. Reformulate the above problem from (6.1)-(6.4) into two different problems by using the $\in$ - constraint method as follows:

Primal problem (1):

$$
\min _{x_{1}} F_{1}(x)=20 x_{1}^{-1} x_{2}^{-3} x_{3}^{-5}+60 x_{1}^{-1} x_{2}^{-1}
$$

s.t.

$$
\begin{aligned}
& 50 x_{1}^{-1} x_{2}^{-2} x_{3}^{-2}+60 x_{1}^{3} x_{2}^{-2} x_{3}^{-3} \leq \epsilon_{2}, \\
& x_{1} x_{2} x_{3}^{2}+x_{2} x_{3} \leq 3 \\
& \left(x_{1}, x_{2}, x_{3}\right)>0 .
\end{aligned}
$$

Primal problem (2):

$$
\min _{x_{1}} F_{2}(x)=50 x_{1}^{-1} x_{2}^{-2} x_{3}^{-2}+60 x_{1}^{3} x_{2}^{-2} x_{3}^{-3}
$$

s.t.

$$
\begin{aligned}
& 20 x_{1}^{-1} x_{2}^{-2} x_{3}^{-5}+60 x_{1}^{-1} x_{2}^{-1} \leq \epsilon_{1}, \\
& x_{1} x_{2} x_{3}^{2}+x_{2} x_{3} \leq 3 \\
& \left(x_{1}, x_{2}, x_{3}\right)>0 .
\end{aligned}
$$

The corresponding Dual program for the primal problem (1) and the Dual program for primal problem (2) is given by (Ojha and Biswal, 2014).

4. Build the membership function $\mu_{F_{1}}, \mu_{F_{2}}$ by using (5.2), and then solve the problem from (5.3) - (5.7) as follows:

$\operatorname{Max} \alpha$

s.t.

$$
20 x_{1}^{-1} x_{2}^{-3} x_{3}^{-5}+60 x_{1}^{-1} x_{2}^{-1}+(49.89122-20.66567) \alpha \leq 49.89122,
$$




$$
\begin{aligned}
& 50 x_{1}^{-1} x_{2}^{-2} x_{3}^{-2}+60 x_{1}^{3} x_{2}^{-2} x_{3}^{-2}+(404.0162-18.20754) \alpha \leq 404.0162 \\
& x_{1} x_{2} x_{3}^{2}+x_{2} x_{3} \leq 3 \\
& \left(x_{1}, x_{2}, x_{3}\right)>0 \quad, \alpha \in[0,1]
\end{aligned}
$$

The optimal compromise solution is obtained as:

$$
\alpha=0.91127,\left(x_{1}^{U}=1.072595, x_{2}^{U}=50157517, x_{3}^{U}=0.4053974\right) \text {. }
$$

The corresponding values of $F_{1}$ and $F_{2}$ are 23.25882 and 52.44017 respectively

Second, Using the steps of algorithm (2) in FPLLMOGPP as:

1. Finds individual optimal solution for each objective functions (6.5) and (6.6) as:

$$
\min _{x_{2}, x_{3}} f_{1}(x)=x_{1}^{-2}+0.25 x_{2}^{2} x_{3}^{-1}
$$

s.t.

$$
\begin{aligned}
& g_{1}(x)=\frac{3}{4} x_{1}^{2} x_{2}^{-2}+\frac{3}{8} x_{2} x_{3}^{2} \leq 1, \\
& \left(x_{1}, x_{2}, x_{3}\right)>0 .
\end{aligned}
$$

Local optimal solution is:

$\min f_{1}(x)=1.171595$ for $\left(x_{1}=1.2395, x_{2}=1.270112, x_{3}=0.774514\right)$.

Also,

$$
\min _{x_{2}, x_{3}} f_{2}(x)=2 x_{1}^{-1} x_{2}^{-1} x_{3}^{-1}+2 x_{1} x_{2}
$$

s.t.

$$
\begin{aligned}
& g_{1}(x)=\frac{3}{4} x_{1}^{2} x_{2}^{-2}+\frac{3}{8} x_{2} x_{3}^{2} \leq 1 \\
& \left(x_{1}, x_{2}, x_{3}\right)>0
\end{aligned}
$$

Local optimal solution is:

$\min f_{2}(x)=3.504279$ for $\left(x_{1}=0.62271, x_{2}=1.205879, x_{3}=1.330079\right)$.

2. Using the local solution of $\left(\mathrm{p}_{5}\right)$ in (6.6) and the local solution of $\left(\mathrm{p}_{6}\right)$ in (6.5) in order to find the lower bound $l_{j}$ and upper bound $u_{j}$ of the function $f_{j}, j=1,2$ as:

$l_{l}=1.171595<f_{1}<2.852155=u_{1} ;$ and $l_{2}=3.504279<f_{2}<4.78887=u_{2}$.

Corresponding $\in_{3}$ and $\in_{4}$ defined by:

$1.171595<\epsilon_{3}<2.85215$; and $3.504279<\epsilon_{4}<4.78887$.

3. Reformulate the problem from (6.5) - (6.8) into two different problems using the $\in$ constraint method as follows:

Primal problem (3):

$$
\min _{x_{2}, x_{3}} f_{1}(x)=x_{1}^{-2}+0.25 x_{2}^{2} x_{3}^{-1}
$$

s.t.

$$
2 x_{1}^{-1} x_{2}^{-1} x_{3}^{-1}+2 x_{1} x_{2} \leq \in_{3}
$$




$$
\begin{aligned}
& \frac{3}{4} x_{1}^{2} x_{2}^{-2}+\frac{3}{8} x_{2} x_{3}^{2} \leq 1, \\
& x=\left(x_{1}, x_{2}, x_{3}\right)>0 .
\end{aligned}
$$

Primal Problem (4):

$$
\min _{x_{2}, x_{3}} f_{2}(x)=2 x_{1}^{-1} x_{2}^{-1} x_{3}^{-1}+2 x_{2} x_{2}
$$

s.t.

$$
\begin{aligned}
& x_{1}^{-2}+0.25 x_{2}^{2} x_{3}^{-1} \leq \in_{4}, \\
& \frac{3}{4} x_{1}^{2} x_{2}^{-2}+\frac{3}{8} x_{2} x_{3}^{2} \leq 1, \\
& x=\left(x_{1}, x_{2}, x_{3}\right)>0
\end{aligned}
$$

The corresponding Dual program for the primal problem (3) and the Dual program for the Primal problem (4) is given by (Ojha and Ota, 2014).

4. Build the membership functions $\mu_{f_{1}}, \mu_{f_{2}}$ by using (5.11), and then solve the problem from $(5.12)-(5.16)$ as follows:

$\operatorname{Max} \beta$

s.t.

$$
\begin{aligned}
& x_{1}^{-2}+0.25 x_{2}^{2} x_{3}^{-1}+(2.852155-1.171595) \beta \leq 2.852155 \\
& 2 x_{1}^{-1} x_{2}^{-1} x_{3}^{-1}+2 x_{1} x_{2}+(4.78887-3.504279) \beta \leq 4.78887 \\
& \frac{3}{4} x_{1}^{2} x_{2}^{-2}+\frac{3}{8} x_{2} x_{3}^{2} \leq 1 \\
& x=\left(x_{1}, x_{2}, x_{3}\right)>0, \quad \beta \in[0,1]
\end{aligned}
$$

The optimal compromise solution as:

$$
\beta=0.780441 \text { for }\left(x_{1}^{L}=0.8932686, x_{2}^{L}=1.135477, x_{3}^{L}=1.121792\right) \text {. }
$$

The corresponding values of $f_{1}$ and $f_{2}$ are 1.5405769 and 3.786322 respectively. Substituting by optimal compromise solution $\left(x_{1}^{L}, x_{2}^{L}, x_{3}^{L}\right)$ in $F_{1}$ and $F_{2}$ respectively, and an optimal compromise solution $\left(x_{1}^{U}, x_{2}^{U}, x_{3}^{U}\right)$ in $f_{1}$ and $f_{2}$ respectively, we get: 23.25886362 < $F_{1}<67.763886$; and 52.44017 $<F_{2}<54.2500931,1.5405769<f_{1}<$ 17.27286341; and $3.786322<f_{2}<11.95566443$

Third, Appling the steps in algorithm (3) we have:

1. Assume the ULMOGPP's control decision $x_{1}^{U}$ with tolerance $k=1$.

2. Using (5.19), (5,20) and (5.21) we get membership function $\mu_{x_{1}}, \mu_{F 1}, \mu_{F_{2}}, \mu_{f_{1}}, \mu_{f_{2}}$, then the LLMOGPP solves the following problem from (5.22) $-(5.30)$ as: $\max \delta$ 


$$
\begin{aligned}
& \text { s.t. } \\
& 20 x_{1}^{-1} x_{2}^{-3} x_{3}^{-5}+60 x_{1}^{-1} x_{2}^{-1}+44.50502251 \delta \leq 67.76388613 \\
& 50 x_{1}^{-1} x_{2}^{-2} x_{3}^{-2}+60 x_{1}^{3} x_{2}^{-2} x_{3}^{-3}+1.8099231 \delta \leq 54.2500931 \\
& x_{1}^{-2}+0.25 x_{2}^{2} x_{3}^{-1}+15.73228651 \delta \leq 17.27286341 \\
& 2 x_{1}^{-1} x_{2}^{-1} x_{3}^{-1}+2 x_{1} x_{2}+8.16934196 \delta \leq 11.95566443 \\
& x_{1}-0.07 \geq \delta \\
& 2.07-x_{1} \geq \delta \\
& x_{1} x_{2} x_{3}^{2}+x_{2} x_{3} \leq 3 \\
& \frac{3}{4} x_{1}^{2} x_{2}^{-2}+\frac{3}{8} x_{2} x_{3}^{2} \leq 1 \\
& x=\left(x_{1}, x_{2}, x_{3}\right)>0, \delta \in[0,1]
\end{aligned}
$$

The compromise solution of BLMOGPP is

$\left(x_{1}=1.071753, x_{2}=2.114856, x_{3}=0.7749304\right)$, and the corresponding values of objective functions are $\quad\left(F_{1}=33.9266984, F_{2}=53.86580161\right.$ $, f_{1}=2.318925741, f_{2}=5.665864011$ ). Also $\delta=0.77$ (overall satisfaction both DMs). Since the ULMOGPP and $\epsilon_{1}$ and $\epsilon_{2}$ are satisfied with the above solution, then a satisfactory solution is reacted.

Generally, combined with set of control variable and objectives with tolerance, the solution of the above problem becomes a Pareto optimal (satisfactory) solution for both DMs.

\section{Conclusion}

This paper has proposed a special class of multi-objective fuzzy bi-level nonlinear problems. This special class is represented in geometric programming problem (GPP) which is a powerful tool for solving some special type nonlinear programming problems. In method of multiobjective geometric programming problem (MOGPP) we have solved one objective function at a time to find the optimal solution and putting other objectives in the constraints by using $\in$ - constraint method. The corresponding fuzzy programming technique of the MOGPP is applied in two levels to give an optimal compromise solution. The solution method uses the concepts of tolerance membership functions at each level to develop a fuzzy max-min decision model for generating Pareto optimal (satisfactory) solution for BLMOGPP. The proposed solution method proceeds from upper level to lower level in a natural and straightforward manner, based on Lai's satisfactory solution concepts. The ULMOGPP specifies his/her objectives and decisions with possible leeway, which are described by membership function of fuzzy set theorem. This information then constrains the LLMOGPP,s feasible space. Also, here we have developed a new algorithm for fuzzy programming technique to solve BLMOGPP to find 
an optimal compromise solution by using $\in$ - constraint method. Finally, an illustrative numerical example has been given to clarify the proposed solution method.

\section{References:}

1. Abo-Sinna, M. A. (2001). A Bi-level Nonlinear Multiobjective Decision Making Under Fuzziness., Sci. Bull. Fac. Eng. Ain Shams Univ.., 36(2).

2. Bard, J. (1999). Practical Bilevel Optimization: Algorithms and Applications., Kluwer Academic Publishers, Boston.

3. Bazikar, F., and Sarj, M. (2014). Solving Linear Multiobjective Geometric Programming Problems Via Reference Point Approach., Sains Malaysiana, 43(8), 1271-1274.

4. Beightler, C. S., and Philips, D. T. (1976). Applied Geometric Programming., Wiley, New York.

5. Bellman, R. E., and Zadah, L. A. (1970). Decision Making in Fuzzy Environment., Mang. Sci., 17B, 141-164.

6. Bit, A.K. (1998). Multiobjective Geometric Programming Problem., Fuzzy Programming with Hyperbotic Membership Function., J. Fuzzy Math., 6, 27-32.

7. Chankong, V., and Haimes, Y. Y. (1983). Multiobjective Decision Making., Theory and Methodology., North - Holand., New York.

8. Duffin, R.J., Peterson, E.L., and Zener, G. (1967). Geometric Programming Theory and Application., John Wiley \& sons., New York.

9. Haimes, Y. Y., Lasdon, L. S., and Wismer, D. A. (1971). On a Bicriterion Formulation of Problems of Integrated System Identification and System Optimization., IEEE Trains. Syst. Man Cybern.., 1(3), 296-297.

10. Hwang, C.L., and Masud, A. (1979). Multiple Objective Decision Making Methods and Applications., A State of Art Survey., Lecture Notes in Economics and Mathematical Systems., Vol. 164, Springer - Variage.

11. Islam, S. (2010). Multiobjective Geometric Programming Problem and its Applications., Journal of Operation Research., Yugoslav., 20(2), 213-227.

12. Islam, S., and Roy, T. K. (2005). Modified Geometric Programming and its Applications., Journal of Applied Mathematics and Computing., Korea., 17(1-2), 121-144.

13. Lee, E. S., and Hsu-Shihshih. (2001). Fuzzy and Multilevel Decision Making., Sprinrger - Verlag London Limited.

14. Lu, J., Han, J., Hu, Y., and Zhang, G. (2016). Multilevel Decision - Making: A survey., Information Sciences., 346 - 347, 463- 487.

15. Mavrotas, G. (2009). Effective Implementation of the $\in-$ Constraint Method in Multiobjective Mathematical Programming Problems., App. Math. Comput., 213, 455-465. 
16. Ojha, A.K., and Biswal, K .K. (2014). Multiobjective Geometric Programming Problem with $\in-$ Constraint Method., Applied Mathematical Modelling., 38, $747-758$.

17. Ojha, A.K., and Ota, R. R. (2014). Multiobjective Geometric Programming Problem with KARUSH - KUHN - TUCKER Condition Using $\in$ - Constraint Method., RAIRO - Operations Research., 48, 429-453.

18. Osman, M. S., Abo-Sinna, M. A., Amer, A. H., and Emam, O. E. (2004). A multilevel Nonlinear Multiobjective Decision Making Under Fuzziness., Appleed Mathemations and Computation, 153, 239-252.

19. Peterson, E. L. (2001). The Fundamental Relations Between Geometric Programming Duality, Parametric Programming Duality and Ordinary Lagrangian Duality., Annal. Oper. Res.., 105, 109-153.

20. Peterson, E.L. (2001). The Origin of Geometric Programming., Annals of Operations Research., 105, 15-19.

21. Sakawa, M. (1993). Fuzzy Sets and Interactive Multiobjective Optimization., Plenum Press, New York.

22. Vicente, L. N., and Calamai, P. H. (1993). Bilevel and Multilevel Programming: A Bibliography Review. Journal of Global Optimization, 3, 291-306.

23. Von Stackelberg, H. (1952). The Theory of the Market Economy., Oxford University Press, Oxford.

24. Yang, J. H., and Cao, B. Y. (2010). Fuzzy Geometric Programming and its Application., Fuzzy Inf. Eng.., 1, 101-112.

25. Zhanf, R. (2003). Multistage Bilevel Programming Problems., Optimization., 52, Nos.4-5, August - October, 605-616.

26. Zhou, X. G., Yang, X. P., and Cao, B. Y. (2016). Posynomial Geometric Programming Problem Subject to max - min Fuzzy Relation Equations., Information Sciences., 328, 15-25.

27. Zimmermann, H. J. (1990). Fuzzy Set Theory and Its Applications., $2^{\text {nd }}$ ed. Kluwer Academic Publishers, Dordrecht-Boston. 